\title{
Atomic Force Microscopy for the Characterisation of the Effects and Treatment of Infectious Parasites
}

P. Eaton*, J.R.S.A. Leite**, C. Bittencourt**, M. Prudêncio***, M.J. Feio*, V. Zuzarte-Luis***, M.M. Mota***, N.C. Santos***, E. Pereira***

*REQUIMTE/Departamento de Química e Bioquímica, Faculdade de Ciências, Universidade do Porto, Porto, Portugal

**Núcleo de Pesquisa em Biodiversidade e Biotecnologia, Biotec, Campus de Parnaíba, Universidade Federal do Piauí, UFPI, Piauí, Brazil

***Instituto de Medicina Molecular, Faculdade de Medicina, Universidade de Lisboa, Portugal

In this talk the utility of atomic force microscopy (AFM) for research into infectious parasites will be discussed. AFM has grown from relatively recent beginnings to become an extremely powerful technique in the life sciences, coupling high resolution imaging with a range of non-imaging experiments. Importantly, these experiments can be performed in situ, even on individual molecules or on live cells [1].

The two examples discussed relate to the important diseases leishmaniasis and malaria. Leishmaniasis is a disease caused by the protozoan parasite of the Leishmania genera, and causes approximately 60,000 deaths per year. Despite the high death toll, the disease has been the subject of relatively little research and little treatment is available, probably because the most severe cases are confined to developing nations. The most severe form, visceral leishmaniasis is caused by the species known as Leishmania infantum (syn. L. chagasi). A promising new anti-leishmania drug, DS01 has been recently isolated from amphibian secretions and can kill L. infantum in low concentrations [2]. We were able to culture and prepare for microscopy L. infantum promastigotes for the first time, as well as to study the effects of DS01 on cell morphology and membrane integrity. The results from both AFM and SEM are highly complementary and illustrate the possibility of membrane-focussed activity as well as the possibility of attack on the flagella (figure 1).

Malaria is one of the most deadly diseases in the world, killing more than 600,000 people per year, mostly in low-income countries. It is caused by Plasmodium parasites, and the most commonly studied stage is that in which the parasite invades the blood. Prior to blood invasion, the parasites infect hepatocytes in the liver, with formation of a parasitophorous vacuole, where they develop into exoerythrocytic forms and multiply to generate thousands of merozoites, later released into the bloodstream and causing disease. However, infection of liver cells, which is clinically silent, is required for disease progression. We studied infection of liver cells by Plasmodium using combined epifluorescence and atomic force microscopy. We observed significant changes in cell morphology as infection progressed (figure 2). Furthermore we made nanoindentation measurements with the AFM, to determine cellular stiffness. We observed stiffening of the cells after 48 hours of infection compared to uninfected cells. This was a cellular response to the Plasmodium infection, rather than a result of the stiffness of the invading parasites themselves. This stiffening may be caused by reinforcement of cytoskeletal structures, and we believe this may reflect a self-defence mechanism by the cell itself [3].

References

1. Eaton P. and West P., Atomic Force Microscopy, OUP: 2010.

2. Zampa M. et al., Nanomedicine: NBM, 5:352-358, 2009.

3. Eaton P. et al., Nanomedicine: NBM, 8:17-19, 2012. 


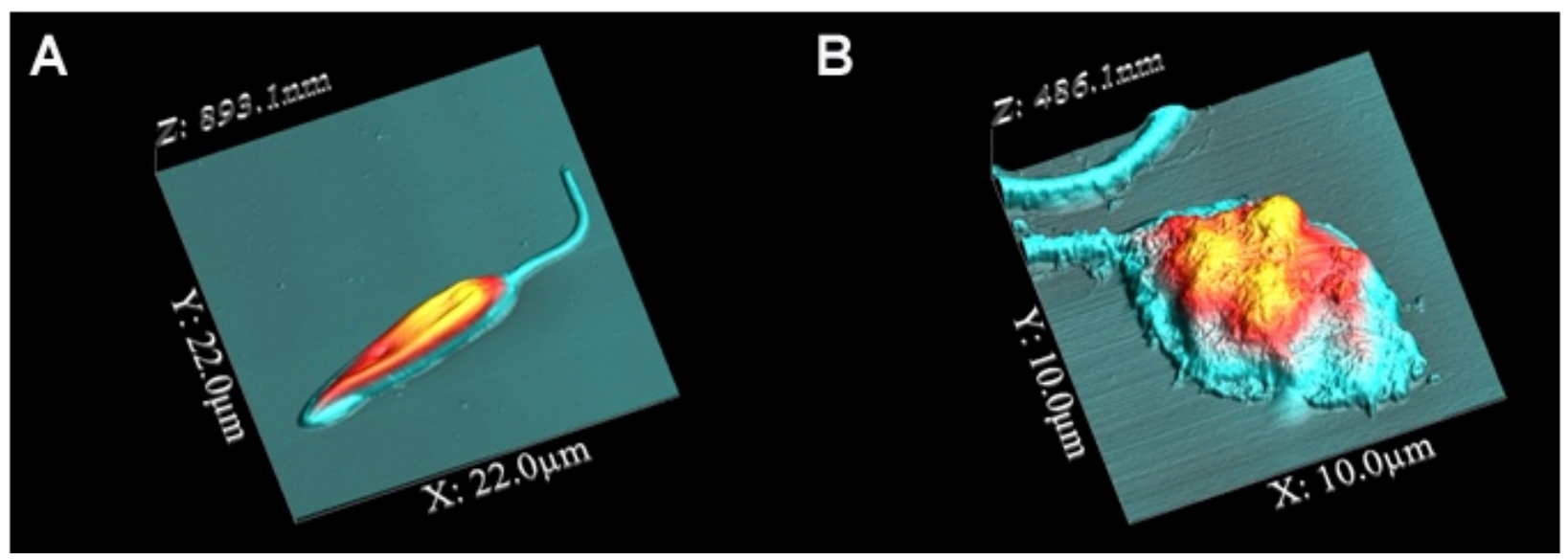

Fig. 1. AFM images obtained from L. infantum cells under different treatment conditions. A: untreated cell showing typical morphology, smooth membrane and flagella. B: Cell treated with the antimicrobial peptide Dermaseptin 01 (DS01) at $128 \mu \mathrm{g} / \mathrm{mL}$, showing membrane disruption, altered morphology and changes in the flagellum.
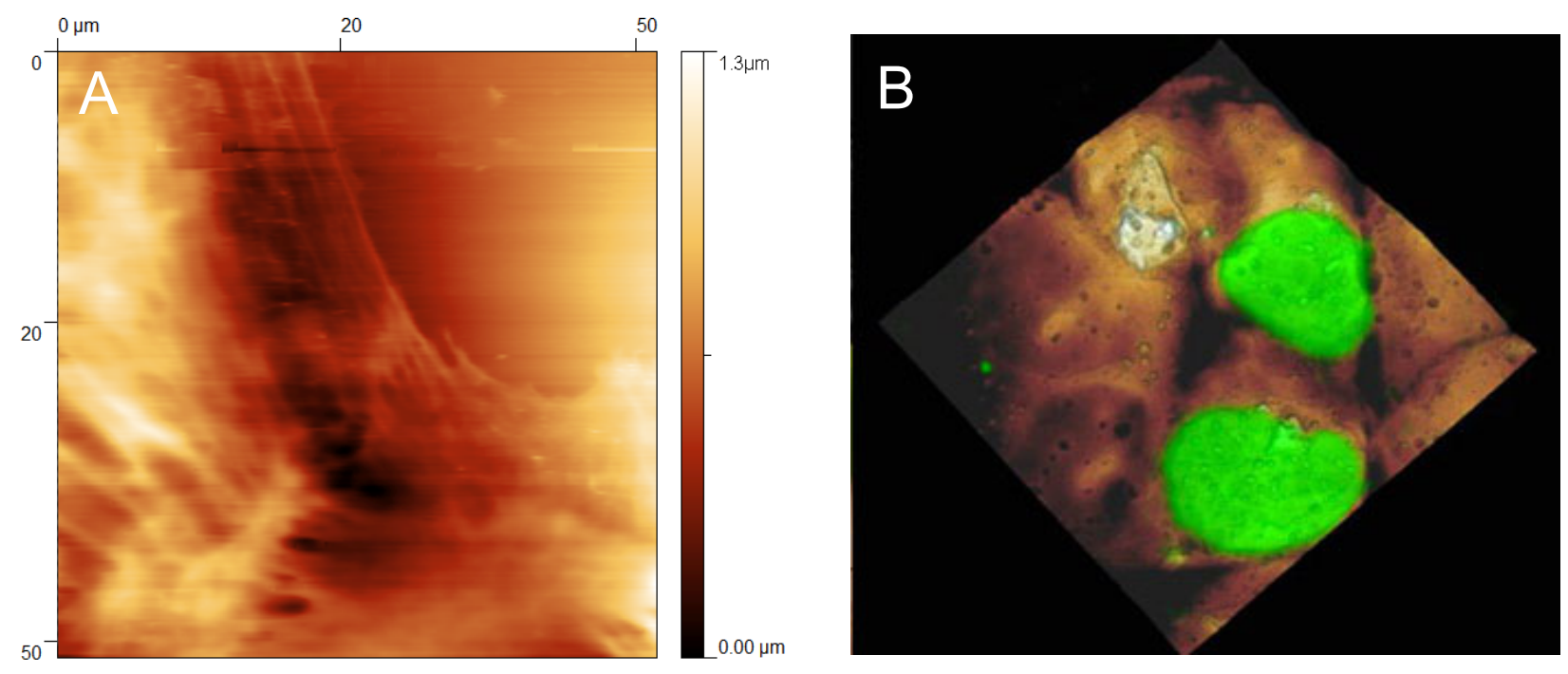

Fig. 2. AFM images obtained from Plasmodium-infected hepatocytes. A: Contact mode images illustrating difficulties in live cell membrane imaging. B: Combined fluorescence and AFM height image of fixed cells showing the locations of two parasitic vacuoles.

The authors acknowledge the funding by Fundação para a Ciência e Tecnologia through grants PTDC/CTMNAN/115125/2009 and PEst-C/EQB/LA0006/2011, and also via a bilateral action between CNPq and FCT. 